\title{
Scaling and root debridement in pregnant women did not result in fewer preterm births
}

\author{
Abstracted from \\ Polyzos NP, Polyzos IP, Zavos A, Valachis A, Mauri D, Papanikolaou EG, Tzioras S, Weber D, Messinis IE. \\ Obstetric outcomes after treatment of periodontal disease during pregnancy: \\ systematic review and meta-analysis. BMJ 2010; 341: c7017 \\ Address for correspondence: Section of Obstetrics and Gynaecology, \\ Panhellenic Association for Continual Medical Research (PACMeR), Athens, Greece. \\ E-mail: n.polyzos@gmail.com
}

\section{Question: Does the treatment of periodontitis and gingivitis during pregnancy reduce the number of preterm births?}

Data sources Cochrane Central, ISI Web of Science, Medline and reference lists of studies; hand searches in key journals.

Study selection Randomised controlled trials (RCTs) in any language that included pregnant women with periodontitis or gingivitis comparing scaling and root planing versus no treatment or prophylaxis. Data extraction and synthesis Two reviewers extracted data and assessed study quality using the Cochrane risk of bias tool. The primary outcome was the rate of preterm births. Odds ratios were calculated using $2 \times 2$ tables and heterogeneity assessed using $\chi^{2}$ and $I^{2}$ tests. Data were synthesised using the fixed effects or random effects models depending on the level of statistical heterogeneity. Studies reporting no events in either arms were excluded. High and low quality studies were synthesised separately. Publication bias was assessed using a funnel plot.

Results Eleven trials (6558 patients) were included, five were considered to be at low, and the rest at high or medium, risk of bias ( 4,718 patients). Meta-analysis found no difference in preterm birth rate using the random effects model (odds ratio $(\mathrm{OR})=0.79,95 \%$ confidence interval $(\mathrm{Cl}) 0.58$ to 1.06 ) or using the fixed effects model ( $\mathrm{OR}=0.93,95 \% \mathrm{Cl} 0.79$ to 1.10$)$. Analysing only the higher quality studies, the lack of difference between interventions was even clearer ( $\mathrm{OR}=1.15,95 \% \mathrm{Cl} 0.95$ to 1.40$)$. There was evidence of publication bias.

Conclusions Scaling and root debridement in pregnant women with periodontitis or gingivitis does not result in fewer preterm births.

\section{Commentary}

This excellent meta-analysis follows a report a year earlier ${ }^{1}$ from the same group. However, that 2009 review came to a different conclusion. In that work they also conducted a meta-analysis of RCTs to determine whether periodontal disease treatment with scaling and/or root planing during pregnancy reduced preterm birth (PTB). They found that treatment resulted in significantly lower PTB (odds ratio [OR], 0.55; 95\% confidence interval $[\mathrm{CI}], 0.35-0.86)$. They noted that 'if ongoing large and well-designed randomised trials support our results, we might need to reassess current practice or at least be cautious prior to rejecting treatment of periodontal disease with scaling and/or root planing during pregnancy.' In contrast, in this review, ${ }^{1}$ they found 'no difference in preterm birth rate between the groups' concluding that such treatment 'cannot be considered to be an efficient way of reducing the incidence of preterm birth.'
What happened in such a short interval of time that led to the differing conclusion, and what lessons may we learn from this? Simply, new findings from large, high-quality trials appeared in the interval. Those trials showed no evidence of a significant protective effect of periodontal treatment. Importantly, when the analysis was restricted to the higher quality studies the lack of difference between treatment arms was even clearer ( $\mathrm{OR}=1.15,95 \% \mathrm{CI} 0.95$ to 1.40$)$. Moreover, this review compared the results from the high quality trials to those from the low quality trials. Not surprisingly, the result from only the low quality trials indicated a protective effect against PTB as well as low birth weight from the periodontal treatment. They also noted publication bias that may have favoured reporting of positive trial results, particularly from smaller studies.

This evident lack of a significant beneficial effect of periodontal treatment should not be taken as proof of the absence of a causal relationship between periodontal infection/inflammation and pregnancy outcomes. The periodontal treatment may have occurred too late in the course of pregnancy and/or the interventions may have been insufficient to reduce the risk. We may, though, view the 'null' finding as added evidence supporting the safety of periodontal treatment during pregnancy. Michalowicz et al. ${ }^{3}$ had earlier reported that 'essential dental treatment ... and/or scaling and root planing, and/or use of topical or local anesthetics during root planing ... was not associated with an increased risk of experiencing serious medical adverse events or adverse pregnancy outcomes.' However, this reassuring conclusion needs to be more widely disseminated. For example, a recent survey of knowledge and beliefs regarding oral health among pregnant women ${ }^{3}$ found that barely half of the women surveyed disagreed with the statement that 'It is not safe for pregnant women to get routine dental care such as checkups and cleanings,' and that Hispanic women were much more likely than white or black women to believe that routine dental care is unsafe during pregnancy. This review is thus timely and should serve as an essential source of high quality evidence to help health professionals to allay such concerns in their patients and to promote optimal oral health throughout pregnancy.

Raul Garcia

Health Policy \& Health Services Research, Henry M. Goldman School of Dental Medicine, Boston University, Boston, MA, USA

1. Polyzos NP, Polyzos IP, Mauri D, et al. Effect of periodontal disease treatment during pregnancy on preterm birth incidence: a metaanalysis of randomized trials. Am I Obstet Gynecol 2009; 200: 225-232.

2. Michalowicz BS, DiAngelis AJ, Novak ], et al. Examining the safety of dental treatment in pregnant women. J Am Dent Assoc 2008; 139: 685-695.

3. Boggess KA, Urlaub DM, Moos MK, Polinkovsky M, El-Khorazaty J, Lorenz C Knowledge and beliefs regarding oral health among pregnant women. / Am Dent Assoc 2011; 142: 1275-1282.

Evidence-Based Dentistry (2011) 12, 112. doi:10.1038/sj.ebd.6400827 